\title{
Aspectos técnicos de los dispositivos de perfusión de órgano aislado
}

\author{
Rodríguez-Martínez D*, Cabello Benavente R, Pedemonte G, Subirá Ríos D, del Cañizo López JF*, \\ Lledó García E.
}

Servicio de Urología. *Unidad de Medicina y Cirugía Experimental. Hospital General Universitario Gregorio Marañón. Madrid.

Actas Urol Esp. 2008;32(1):59-66

\section{RESUMEN}

ASPECTOS TÉCNICOS DE LOS DISPOSITIVOS DE PERFUSIÓN DE ÓRGANO AISLADO

Un aspecto de gran importancia en el campo de los trasplantes es el mantenimiento y preservación del órgano durante la fase isquémica. La sensibilidad del órgano a ésta y la posible lesión inducida durante la extracción, preservación y transporte, siguen siendo una de las principales causas de fracaso del injerto. La perfusión artificial de órganos ha sido objetivo de investigación durante más de 100 años. En la segunda mitad del siglo XX estas técnicas han sido aplicadas a la preservación experimental y clínica, con el fin de alargar el tiempo de almacenamiento efectivo (aquél que permite una función correcta y precoz tras el trasplante del órgano preservado). La meta primaria de la preservación de cualquier órgano es el mantenimiento de la integridad de todos los sistemas celulares, de tal manera que la posibilidad de lesión que lleve a la disfunción del injerto sea mínima.

Palabras clave: Perfusión renal. Preservación de órganos.

\section{ABSTRACT \\ TECHNICAL ASPECTS OF ISOLATED ORGAN PERFUSION DEVICES}

An aspect of capital importance in the transplant field is the maintainance and preservation of organs during the ischemic phase. Organ susceptibility and injuries secondary to ischemia, organ procurement and transportation are still nowadays one of the main causes for organ failure. Effective artificial organ perfusion has remained a main goal for researchers in this field for more than 100 years. In the second half of the XXth century these techniques have been applied to experimental and clinical organ preservation, with the aim of extending the period of an effective storage (which guarantees an early and good function after the transplant). Primary goal of any organ preservation is to maintain integrity in every cell system in order to minimize those injuries which produces graft dysfunction.

Keywords: Renal perfusion. Organ preservation.

$\mathrm{U}$ aspecto de gran importancia en el campo de los trasplantes es el mantenimiento y preservación del órgano durante la fase isquémica. La sensibilidad del órgano a ésta y la posible lesión inducida durante la extracción, preservación y transporte, siguen siendo una de las principales causas de fracaso de injerto. La perfusión artificial de órganos ha sido objetivo de investigación durante más de 100 años. En la segunda mitad del siglo
XX estas técnicas han sido aplicadas a la preservación experimental y clínica, con el fin de alargar el tiempo de almacenamiento efectivo (aquél que permite una función correcta y precoz tras el trasplante del órgano preservado). La meta primaria de la preservación de cualquier órgano es el mantenimiento de la integridad de todos los sistemas celulares, de tal manera que la posibilidad de lesión que lleve a la disfunción del injerto sea mínima. 


\section{EVOLUCIÓN HISTÓRICA DE LA PERFUSIÓN DE ÓRGANO AISLADO}

La perfusión artificial de órganos ha sido objetivo de investigación durante más de 100 años. En la segunda mitad del siglo XX estas técnicas han sido aplicadas a la preservación experimental y clínica, con el fin de alargar el tiempo de almacenamiento efectivo (aquél que permite una función correcta y precoz tras el trasplante del órgano preservado).

La primera referencia bibliográfica encontrada en la literatura data de 1812 cuando J.J. Cesar-Le Gallois ${ }^{1}$ plantea la sustitución del corazón por un sistema de bombeo que, de forma continua proporcionase sangre arterial o un medio líquido alternativo adecuado para mantener viable de forma indefinida una parte aislada del organismo. Esta afirmación regiría la mayoría de avances posteriores. Leobel1 ${ }^{2}$ parece ser el primero en realizar una perfusión renal experimental. En la segunda mitad del siglo XIX se llevan a cabo experiencias sobre sistemas de oxigenación sanguínea para perfusión renal aislada ${ }^{3,4}$. Finalmente $\mathrm{Jacobi}^{5}$, diseña en 1890 un aparato muy complejo para perfusión renal que oxigena sangre mediante infusión directa de aire, extrayendo posteriormente las burbujas a través del paso de esa sangre a través de un reservorio de estructura helicoidal.

Durante la primera mitad del siglo XX los aspectos dinámicos y bioquímicos de la perfusión de órgano aislado despertaron gran interés. En 1910 Guthrie $^{6}$ concluye que la perfusión puede resultar nociva para el riñón independientemente de la composición del líquido utilizado. Hooker ${ }^{7}$ afirma que la magnitud del pulso de presión aplicado durante la perfusión renal correlaciona con el flujo sanguíneo conseguido y la cantidad de orina formada. En 1913 Gesell $^{8}$ destaca las dificultades que plantea diseñar un sistema experimental de perfusión de órgano aislado. Este autor realiza sus trabajos de perfusión renal en el animal intacto, utilizando modelos con presiones de perfusión pulsátil y no pulsátil. Al contrario que Hooker concluye que la magnitud del pulso de presión no influye en el flujo sanguíneo conseguido aunque sí en el volumen producido de orina.
Los diseños experimentales para perfusión cardiaca supusieron una fuente importante de investigación e información. Sobresalen en esa época los trabajos de Knowlton y Starling9 quienes describen en 1912 un aparato para autoperfusión sanguínea miocárdica que permitía la determinación del gasto cardiaco y la resistencia periférica. Este sistema, conocido como preparación de Starling ofrecía la posibilidad de mantener corazones de mamíferos latiendo activamente durante varias horas, lo que contribuyó a ampliar el conocimiento sobre la acción del miocardio. Starling utilizó también su preparación para perfusión renal aislada, lo que le permitió estudiar la secreción de orina ${ }^{10}$. Bainbridge y Evans ${ }^{11}$ utilizaron este modelo observando que la presión arterial de perfusión, flujo sanguíneo y temperatura eran controlables, pudiendo estudiarse el órgano bajo condiciones comparables a las del animal vivo. Estos autores confirmaron que el riñón perfundido de esta forma podía producir orina, y que su metabolismo gaseoso no se alteraba por los cambios o redistribución del flujo a través del órgano.

Un hallazgo importante para el desarrollo de posteriores dispositivos de perfusión es el obtenido por Richards y Plant ${ }^{12,13}$, quienes detectan variaciones en la cantidad y composición de la orina producida al modificar la presión sanguínea renal durante la perfusión. Este dato es particularmente importante, puesto que permite pensar que no sólo los factores bioquímicos sino también los dinámicos influyen en el riñón perfundido desde el punto de vista funcional.

La perfusión de órganos se desarrolla en esa época juntamente con el cultivo de tejidos e incluso de órganos enteros aislados. El gran problema con el que se enfrenta esta técnica es la esterilidad del medio. Uno de los investigadores que más trabajó en ese campo fue Alexis Carrel. En 1930 uno de sus colaboradores ${ }^{14}$ describe una bomba magnética acoplada a una cámara de oxigenación para perfusión de riñones; sin embargo este sistema fracasa debido probablemente a contaminación bacteriana. El propio Carrel diseñó junto a Charles Lindbergh un sistema cerrado constituido por una campana de vidrio a la que se adaptaba un aparato de perfusión de líquido en el cultivo, en el que se podian mantener condiciones de esterilidad 
(Lindbergh-Rockefeller Institute Organ Perfusion System) ${ }^{15,16}$. Este dispositivo mantenía la presión de perfusión renal a través de la propia columna de líquido, que circulaba continuamente. Los problemas que hallaron los autores fueron hidrodinámicos, ya que la presión ejercida por el fluido no era suficiente y además no era pulsátil. Sin embargo sí fueron capaces de demostrar ausencia de contaminación bacteriana después de un mes completo de circulación experimental del líquido a través de arteria carótida.

El gran impulsor de la perfusión de órganos fue Belzer. En su trabajo de 1967 describió la posibilidad de realizar perfusión de riñones aislados de manera experimental durante al menos 24 horas, señalando que el daño celular que se produce como consecuencia de un período de preservación es pequeño y rápidamente reversible ${ }^{17}$.

Posteriormente, se definieron dos campos de estudio en la perfusión renal, la conservación por simple hipotermia y la perfusión mecánica del órgano. Los trabajos de autores como Cerra ${ }^{18}$ junto con diversas causas como el perfeccionamiento de las técnicas de extracción de órganos en donante vivos, introducción de soluciones para preservación mediante inmersión hipotérmica simple, el alto precio de los sistemas de perfusión existentes, hicieron que ganara gran interés la conservación por simple hipotermia frente a la perfusión mecánica en bomba.

A pesar de esto, se siguieron estudiando y comparando los dos métodos fundamentales de conservación. Aparecieron numerosos estudios en los que quedaba demostrada la utilidad de la perfusión mecánica sobre la conservación en hipotermia simple ${ }^{19-21}$. Estos estudios, junto con el renovado interés en la obtención de órganos provenientes de donantes en asistolia permitió rescatar las ventajas que podía ofrecer la perfusión continua hipotérmica para disminuir los efectos de la isquemia caliente inicial prolongada sobre el epitelio tubular y el endotelio vascular renal así como determinar el grado de viabilidad del órgano.

Son muchos los trabajos que han aparecido a favor de la perfusión de órganos de donantes a corazón parado frente a la conservación en hipotermia simple ${ }^{22,23}$ haciendo de la perfusión mecánica en bomba el método de elección de conservación de órganos en la actualidad.

\section{CARACTERÍSTICAS GENERALES DE LOS DISPOSITIVOS DE PERFUSIÓN DE ÓRGANO AISLADO}

En general todos los sistemas de perfusión tienen una serie de factores comunes: una bomba que hace circular la solución de perfusión a través del órgano, un reservorio donde se encuentra el líquido y un sistema de refrigeración y un sistema de control que controla todo el dispositivo; algunos de ellos poseen un oxigenador. Cuentan además con una serie de sensores que permiten el registro de los parámetros hemodinámicos de flujo y de presión de perfusión.

De todos los elementos que componen los dispositivos, el componente diferenciador entre unos y otros va a ser la bomba de impulsión de líquido.

\section{Bomba de perfusión}

El tipo de bomba que va a existir en el dispositivo va a ser seleccionada en función del tipo de flujo con el que se quiera dotar al mismo. Básicamente, las bombas que se utilizan pueden proporcionar dos patrones de flujo bien diferenciados: flujo pulsátil o flujo continuo.

Independientemente del flujo que proporcionen, los sistemas de bombeo se pueden clasificar en tres tipos fundamentales:

- Peristálticas

- Rotativas

- De desplazamiento

Bombas peristálticas: Se basan en la compresión progresiva de un tubo elástico. Las más conocidas son las bombas de rodillos que se utilizan habitualmente en circulación extracorpórea (CEC) y hemodiálisis (Fig. 1). Su funcionamiento es muy sencillo, un motor eléctrico mueve un cabezal provisto de dos a más rodillos que comprimen un tubo elástico contra una carcasa rígida. Tienen el inconveniente de su gran tamaño y el alto nivel de hemólisis que producen lo que limita su utilización a unas horas. Generan flujo pulsátil.

Bombas rotativas: Su funcionamiento se basa en el giro de un rotor, producen, por tanto, flujo continuo; existen dos tipos fundamentales:

\section{- Bombas axiales}




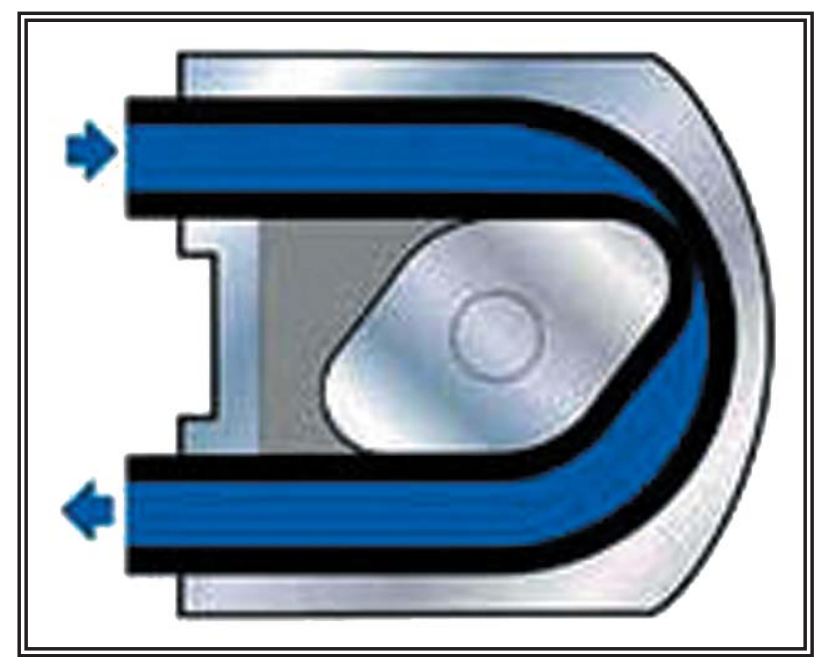

FIGURA 1. Esquema de una bomba peristáltica de rodillos.

- Bombas centrifugas: Constituidas por una cámara de forma más o menos cónica con un tubo de salida tangencial y un tubo de entrada central. En el interior de esta cámara se mueve un rotor que hace que el líquido gire a gran velocidad y sea impulsada, gracias a la fuerza centrífuga, por el tubo de salida (Fig. 2).

Las bombas rotativas producen menor hemólisis que las bombas de rodillos y algunas de ellas están diseñadas para asistencias de larga duración. Proporcionan flujo continuo y no requieren válvulas.

Son de tamaño pequeño, su funcionamiento es sencillo y se limita a controlar la velocidad de giro del rotor, el consumo de energía es bajo, son silenciosas.

Bombas de desplazamiento: Tratan de imitar el funcionamiento normal del corazón, producen flujo pulsátil y están provistas de válvulas de entrada

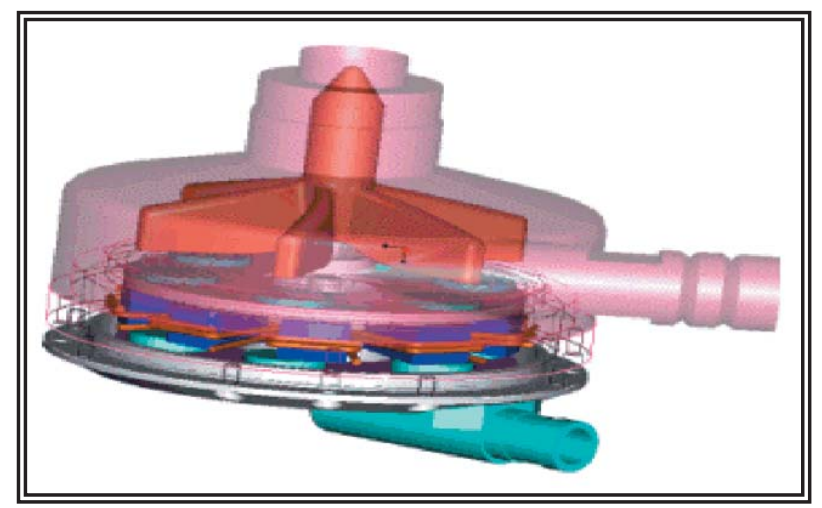

FIGURA 2. Esquema de una bomba rotativa centrifuga. y de salida. La cámara sanguínea se dilata y se comprime en cada latido aspirando el líquido a través de la válvula de entrada e impulsándola hacia la válvula de salida (Fig. 3).

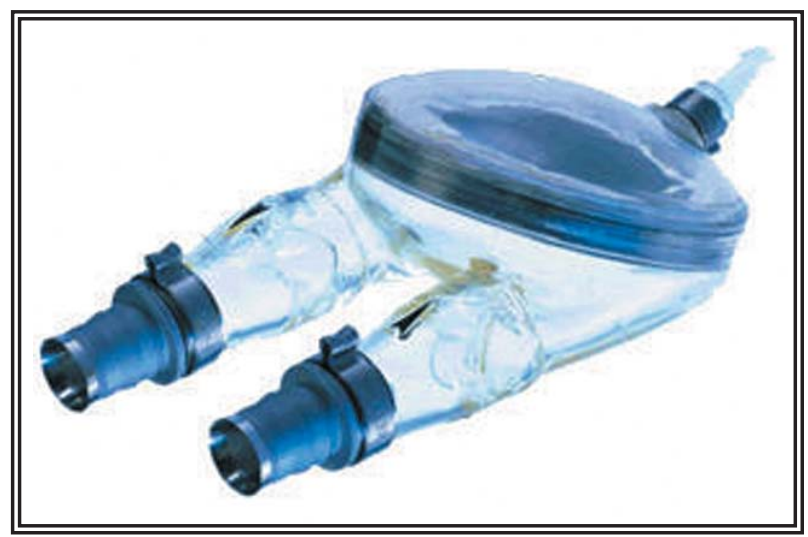

FIGURA 3. Fotografia de una bomba de desplazamiento.

Dependiendo de la forma de la cámara sanguínea pueden ser: De diafragma, Saculares y Tubulares. Dependiendo del mecanismo utilizado para comprimir la cámara pueden ser: Neumáticas o Electromecánicas y, dependiendo de su colocación pueden ser: Paracorpóreas o Intracorpóreas.

El flujo es muy poco dependiente de la precarga y la poscarga, su coste es más bajo que las bombas rotativas, hay una gran experiencia clínica con ellas y no se produce flujo retrógrado si se detienen. Su tamaño es grande, el consumo de energía es alto y son ruidosos.

La diferencia fundamental entre flujo pulsátil y flujo no-pulsátil estriba en la continuidad del flujo, mientras que en el primero se produce una onda de pulso cuya morfología varía dependiendo del sistema intentando simular un flujo fisiológico, en el segundo el flujo es continuo. Además el flujo pulsátil es dependiente de la presión, del volumen de eyección y de la frecuencia de pulso, mientras que en la no-pulsátil el flujo es única y directamente proporcional a la presión de perfusión. Las diferencias existentes dentro del flujo pulsátil dependen de la morfología de la onda de pulso, no estando descrita en la mayoría de los trabajos que utilizan este tipo de flujo.

Aún cuando existen detractores del flujo pulsátil, son numerosos los trabajos que describen sus beneficios. Algunos estudios destacan la impor- 
tancia del flujo pulsátil en modelos de perfusión extracorpórea sistémica, como aplicación en técnicas de asistencia mecánica circulatoria, habiéndose descrito un aumento significativo en el flujo vascular renal tras perfusión extracorpórea pulsátil ${ }^{24}$. Analizando los efectos del bombeo pulsátil a bajo flujo en hipotermia profunda en la perfusión tisular y en la función renal, se observa que cuando la perfusión se realiza con flujo pulsátil, al volver a la temperatura fisiológica, los riñones de los animales que fueron perfundidos con este flujo mostraban mayor producción urinaria, así como, un mayor flujo linfático con menor edema intersticial.

\section{Sistema de refrigeración}

Actualmente, se admiten los beneficios de la perfusión hipotérmica frente a la normotérmica. Por eso, la mayoría de los dispositivos de perfusión mecánica la realizan en condiciones de hipotermia.

Para conseguir estas temperaturas se utilizan los sistemas de refrigeración, consisten en generadores de frío cuya única función es mantener una temperatura adecuada (alrededor de $4^{\circ} \mathrm{C}$ ) en el líquido de perfusión.

\section{Oxigenador}

Algunos sistemas de perfusión intercalan en su circuito un oxigenador para oxigenar el liquido de perfusión.

\section{Sensores de registro de parámetros de perfusión}

Son sensores que van a permitir controlar y saber como varian distintos parámetros (flujo, presión, temperatura) durante la perfusión. Suelen existir tres tipos de sensores:

1. Transductor de flujo: permite conocer el flujo de la perfusión.

2. Transductor de presión: permite conocer la presión de perfusión.

3. Sonda de temperatura: permite saber la temperatura del líquido de perfusión.

\section{Sistema de control}

Sistema que integra todas las señales que 1legan del sistema (transductores) y va a permitir controlar distintos parámetros del dispositivo.

\section{SISTEMA DE PERFUSIÓN HIPOTÉRMICA BASADO EN UNA BOMBA TUBULAR PULSÁTIL GOBERNADA POR VACÍO}

Este sistema de perfusión ha sido diseñado integramente por nuestro grupo en el Laboratorio de la Unidad de Medicina y Cirugía Experimental del Hospital Gregorio Marañón de Madrid ${ }^{25,26}$.

\section{Bomba de perfusión}

El sistema de perfusión se basa en la utilización de una bomba tubular pulsátil no oclusiva gobernada por vacío desarrollada en nuestro laboratorio ${ }^{27}$. Genera una onda de flujo pulsátil similar a la generada por el corazón. Consta de dos cámaras: la cámara de impulsión es un tubo elástico de paredes finas que se encuentra en el interior de una cámara rígida en la cual es posible ejercer vacío gracias a una conexión con una consola de mando. El vacío aplicado a la cámara tubular hace que esta se dilate produciéndose el llenado de la cámara. Cuando se interrumpe el vacío y la cámara rígida se conecta a la presión atmosférica la recuperación elástica de la membrana provoca la impulsión del líquido.

Las válvulas son neumáticas de pinza colocadas en las cánulas de entrada y salida de la bomba.

En la Figura 4 se representa una sección transversal del dispositivo. La conexión para el vacío conecta el espacio estanco que existe entre la membrana y la carcasa con una consola de mando.

Durante el llenado de la bomba, la consola de mando realiza una succión a través de la conexión provocando la expansión de la membrana, al mismo tiempo da la orden de cierre de la válvula de salida y la de apertura de la de entrada con lo que el líquido penetra a través de la cánula de

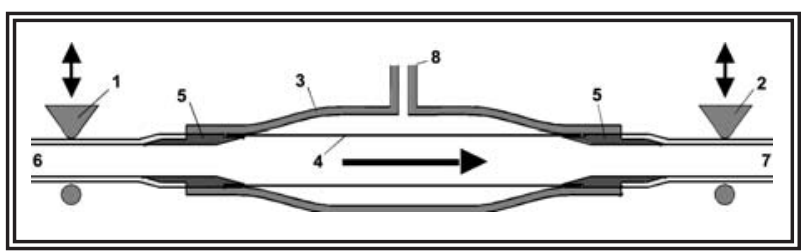

FIGURA 4. Sección transversal de la bomba tubular. 1) Válvula de pinza de entrada. 2) Válvula de pinza de salida. 3) Carcasa rígida. 4) Membrana tubular elástica. 5) Piezas de conexión de las cánulas. 6) Cánula elástica de entrada. 7) Cánula elástica de salida. 8) Conexión para el vacio. 
entrada llenando la cámara (Fig. 5 a y b). Durante la impulsión, la consola de mando conecta la conexión de vacío con la atmósfera, al mismo tiempo da la orden de cierre a la válvula de entrada y la de apertura a la de salida, en ese momento la recuperación elástica de la membrana, liberada del vacío ejercido durante el llenado, impulsa el líquido a través de la cánula de salida (Fig. 5c).

La bomba tubular utilizada en este dispositivo (Fig. 6) tiene algunas ventajas sobre las bombas que suelen estar presentes en otros dispositivos (bombas de rodillos, principalmente):

1. Es una bomba que genera un flujo pulsátil similar al fisiológico, como los ventrículos artificiales, con las ventajas que esto aporta desde el punto de vista fisiopatológico, pero con un coste sensiblemente inferior.

2. El vacío aplicado a la cámara tubular hace que ésta se dilate de forma homogénea sin que existan arrugas que puedan favorecer la formación de agregados plaquetarios y coágulos.

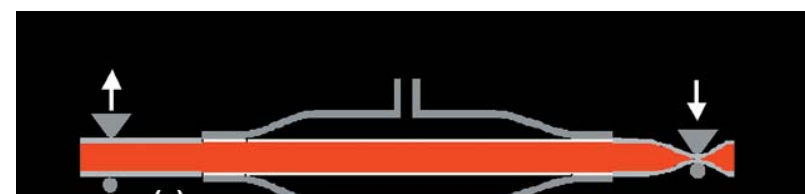

(a)

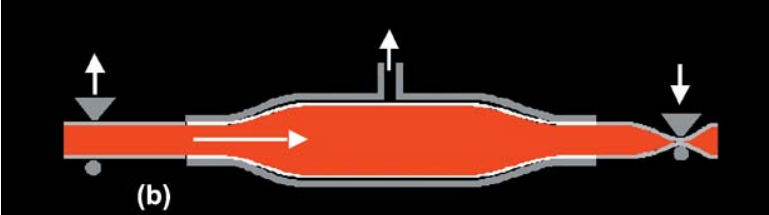

(b)

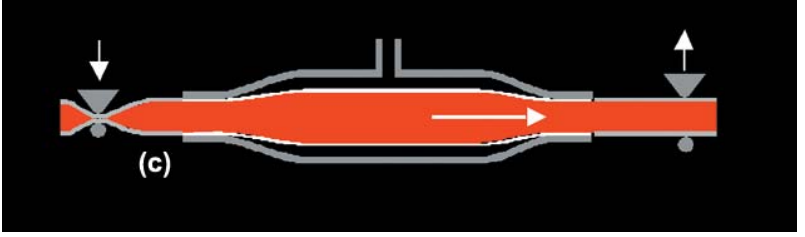

FIGURA 5. a y b) Bomba de vacío - Diástole: cierre válvula de salida, apertura válvula de entrada aplicación de vacio (expansión membrana); c) Bomba de vacio - Sistole: apertura válvula de salida, cierre válvula de entrada, aplicación de presión atmosférica (retorno de membrana).

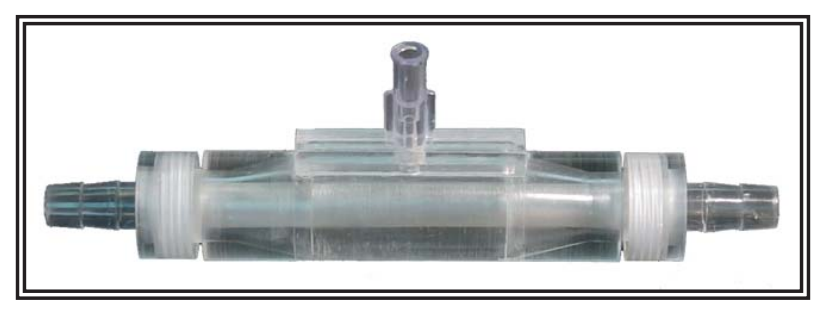

FIGURA 6. Bomba Tubular Pulsátil gobernada por vacio.
3. La utilización exclusiva de vacío hace que en caso de perforación de la membrana, nunca entre gas en el interior de la cámara flexible ya que la presión en ésta es siempre superior a la de la cámara externa, evitándose la posibilidad de que llegase aire al órgano perfundido.

4. La bomba tubular no es oclusiva y la máxima presión que puede generar está limitada por las características de la membrana, por tanto una obstrucción en la salida o en la entrada lo único que provoca es el paro de la bomba sin que en ningún caso pueda destruirse el circuito.

5. En las aplicaciones con oxigenadores se pueden diseñar bombas cuya presión máxima de trabajo sea inferior a la presión de rotura de estos dispositivos con lo cual será imposible le destrucción de los mismos y no se necesitan sistemas de seguridad que paren la bomba en caso de sobrepresión.

6. La bomba utiliza válvulas de pinza sobre las cánulas, por tanto la parte desechable es fácil de construir y muy barata. Este tipo de válvulas hace además que la bomba pueda ser muy pequeña y las cánulas muy finas.

7. La bomba tubular evita los problemas de ajuste de la oclusión de los rodillos y posibles lesiones al manejarla.

8. El aislamiento eléctrico es mucho mayor ya que la bomba está unida a la consola únicamente por un tubo de plástico.

9. Los periodos de aspiración y de impulsión de líquido pueden regularse de modo independiente e incluso separarse en el tiempo, reduciendo la recirculación.

\section{Sistema de refrigeración}

Utilizaremos un generador de frío (Unidad Refrigeradora Frigedor, Lambra S.L.), que dispone de un espiral metálico que se coloca en un baño de agua externo a la cámara de órgano, manteniendo este medio a $2,8-3,2^{\circ} \mathrm{C}$. Esto permite que la temperatura del interior de la cámara de órgano permanezca en un rango constante de $3,8^{\circ} \mathrm{C}$ $4,2^{\circ} \mathrm{C}$, lo que se comprueba mediante medición. La activación y desactivación del aparato de frío es controlada por el ordenador mediante retroinformación suministrada al sistema desde el baño de agua a través de una sonda de temperatura (temperatura $<2,8^{\circ} \mathrm{C}$ se desactiva, $>3,2^{\circ} \mathrm{C}$ se activa). 
Gracias a este control podemos mantener una temperatura con oscilación conocida en el órgano, teniendo en cuenta que el valor permanece constante en la solución contenida en el interior de la cámara, produciéndose sólo discretas pérdidas en el trayecto de la solución de perfusión a través del circuito.

\section{Sensores de registro de parámetros de perfusión}

Se registran los siguientes parámetros:

- Flujo de perfusión: mediante un transductor de flujo por ultrasonidos colocado en el circuito de líquido.

- Presión de perfusión: mediante transductores de presión desechables.

- Sonda de temperatura: colocada en el interior de la cámara donde está el líquido de perfusión.

Todos estos parámetros son enviados a la unidad de control, que va a permitir registrarlos en tiempo real y analizar la calidad de dicha perfusión, viendo como evolucionan distintos parámetros como son: el flujo, la presión y la resistencia vascular renal (calculada como cociente entre la presión y el flujo de perfusión).

\section{Unidad de control}

Consta de dos partes diferenciadas. Una es la parte de registro que nos va a permitir visualizar y almacenar los datos de la perfusión (flujo, presión y resistencia vascular) en tiempo real (Fig. 7).

Por otra parte, tenemos la parte de control de la bomba y del sistema de refrigeración. Permite variar distintos parámetros de funcionamiento de

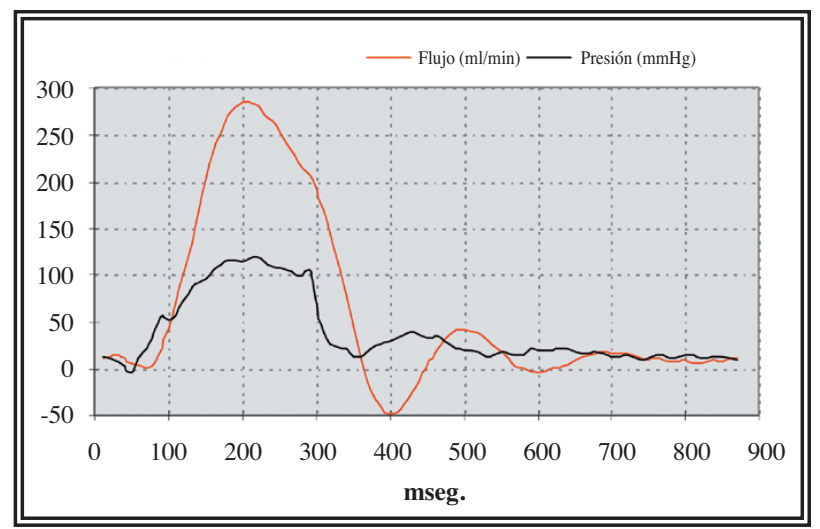

FIGURA 7. Registro de ondas instantáneas de presión flujo en bomba de vacio. la bomba, dependiendo de la evolución de la perfusión, como son: frecuencia (latidos por minuto), duración de la sístole y vacío aplicado durante la diástole. La variación de estos va a permitir cambiar las condiciones de flujo y presión de la perfusión.

En cuanto a la temperatura, esta unidad es capaz de activar o desactivar el generador de frío dependiendo de la temperatura que se esté registrando en el líquido de perfusión.

\section{REFERENCIAS}

1. Le Gallois JJC. Experiénces sur le principe de la vis, notamment sur celui des mouvements du coeur, et sur le siège de ce principe. D`Hautel, Paris, 1812.

2. Leobell L. Dissertation, Marburg (1849), citado en: Starling $\mathrm{EH}$, Verney EB. The secretion of urine as studied on the isolated kidney. Proc Roy Soc. 1925; 97:321-363.

3. Bunge G, Schmiedeberg O. Über die bildung der huppursaur. Arch Exptl Pathol Pharmakol. 1876;6:233-255.

4. Von Schroeder W. Über die bildungsstatte des hanrstoffs. Arch Exptl Pathol Pharmakol. 1885;15:364-402.

5. Jacobi C. Apparat zur Durchbluttung isolirter überlebender organe. Arch Exptl Pathol Pharmakol. 1890;26:388400.

6. Guthrie CC. The effect on the kidney of the temporary anemia, alone and accompanied by perfusion. Arch Internal Med. 1910;5:232-245.

7. Hooker DR. The perfusion of the mammalian medulla: the effect of calcium and of potassium on the respiratory and cardiac centers. Am J Physiol. 1915;38:200-208.

8. Gesell RA. On the relation of pulse pressure to renal secretion. Am J Physiol. 1913;32:70-93.

9. Knowlton FP, Starling EH. The influence of variations in temperature and blood pressure on the performance of the isolated mammalian heart. J Physiol. 1912;44(3):206219.

10. Starling EH, Verney EB. The secretion of urine as studied on the isolated kidney. Proc Roy Soc. 1925;97:321-363.

11. Bainbridge FA, Evans CL. The heart, lung, kidney preparation. J Physiol. 1914;48(4):278-286

12. Richards AN, Plant $\mathrm{OH}$. Urine production in the perfused kidney: The influence of adrenalin on the volume of the perfused kidney. Am J Physiol. 1922;59:184-190.

13. Richards AN, Plant OH. The action of minute doses of adrenalin and pituitrin on the kidney. Am J Physiol. 1922; 59:191-202.

14. Rosenberger H. An electromagnetic pump. Science. 1930; 71(1844):463-464

15. Carrel A, Lindbergh CA. The culture of whole organs. Science. 1935; 81(2212):621-623.

16. Lindbergh CA. An apparatus for the culture of whole organs. J Exptl Med. 1935;62:409-431.

17. Belzer FO, Ashbi BS, Dunphy JE. 24-hour and 72-hour preservation of canine kidneys. Lancet. 1967;2(7515): 536539.

18. Cerra FB, Raza S, Andres GA, Siegel JH. The endotelial damage of pulsatile renal preservation and its relationship to perfusion pressure and colloid osmotic pressure. Surgery. 1977;81(5):534-541. 
19. Southard JH, van Gulik TM, Ametani MS, Vreugdenhil PK, Lindell SL, Pienaar BL, et al. Important components of the UW solution. Transplantation. 1990;49(2):251-257.

20. Ploeg RJ, Goossens D, McAnulty JF, Southard JH, Belzer FO. Succesful 72-hour cold storage of dog kidneys with UW solution. Transplantation. 1988;46(2):191-196.

21. Yland MJ, Nakayama Y, Abe Y, Rapaport FT. Organ preservation by a new pulsatile perfusion method and apparatus. Transpl Proc. 1995;27(2):1879-1882.

22. Booster MH, Yin M, Stubenitski BM, Heidendal GAK, van Kroonenburg MJPG, Kemerink GJ, Hinders SGEA, Heineman E, Buurmen WA, Wijnen RMH, Tiebosch ATM, Bonke H, Kootstra G. Beneficial effect of machine perfusion on the preservation of renal microcirculatory integrity in ischemically damaged kidneys. En Booster MH: The Non-Heart-Beating Donor, a new source for kidney transplantation. Universitaire Pers Maastricht Ed. Maastricht, 1995.

23. Matsuno N, Sakurai E, Tamaki I, Furuhashi K, Saito A, Zhang S, et al. Effectiveness of machine perfusion preservation as a viability determination method for kidneys procured from non-heart-beating donors. Transplant Proc. 1994;26(4):2421-2422.
24. Mandelbaum I, Berry J, Silbert M, Burns WH, Rothe C. Regional blood flow during pulsatile and nonpulsatile perfusion. Arch Surg. 1965;91(5):771-774.

25. del Cañizo JF, Tejedor A, Lledó E, Radvan J, Desco M, Dulin $\mathrm{E}$, et al. Isolated kidney controlled perfusion with true physiological pulsatile waveform. Artif Organs. 1998;22(4):279-284.

26. Herrera B, Eisenberg G, Desco MM, Holberndt O, Rábano A, Castilla M, et al. Perfusate lactate Dehydrogenase level and intrarenal resistance could not be adequate markers of perfusion quality during isolated kidney perfusion. Artificial Organs. 2000;24(11):899-902.

27. del Cañizo JF, Antoranz JC, Duarte J, Desco MM, GarcíaBarreno P. Tubular vacuum-powered blood pumping device with active valves. Artif Organs. 1996;20(7):789-793.

Correspondencia autor: Dr. D. Rodríguez-Martínez Unidad de Medicina y Cirugía Experimental Hospital General Universitario Gregorio Marañón Dr. Esquerdo 46 - 28007 Madrid

Tel.: 914265015

E-mail autor: danielrod@mce.hggm.es Información artículo: Original 